considered the great medical advances of the eighteenth century, of which he mentioned the three most important. Undoubtedly the greatest was the combat with scurvy since scurvy was the scourge of the navy and mercantile marine. Scurvy, he thought, was far more common than was generally supposed; apart from well-marked scurvy many people had the scorbutic taint. In the sixteenth and seventeenth centuries the poor got very little fresh meat and vegetables, living principally on salted meat and fish; cabbage gardens were then by no means common and the potato had not been generally introduced into the natural diet. In 1780 the potato came into the dietary of St. Bartholomew's Hospital. Dr. Church, the present President of the Royal College of Physicians of London, was of opinion that the constant use of cow-dung in the pharmacopceias of former times was possibly due to the fact that grass cooked in this rather unsavoury way was good for the scurvy; there must be something in that for it was hardly conceivable that people would have taken such stuff if they had not got some real benefit. When Commodore Anson made a tour of the world in 1740 he lost nearly the whole of his crew from scurvy; when Cook went round the world in 1769 not a man was lost from this disease, so that somewhere between 1740 and 1780 scurvy was got rid of. Sweet-wort (now extract of malt) was said to have prevented scurvy among Cook's crew; and Sir John Pringle-a great hygienist who had control over the army and was President of the Royal Society-in presenting Cook with the Copley medal complimented him on not having his crew decimated by scurvy. Sic Gilbert Blane, physician to the fleet, ordained that limejuice should be used habitually and freely as a regular article of diet among sailors, and since this introduction the disease had almost disappeared. We see then that we got rid of scurvy by our advance in agriculture and horticulture.

The detection of the cause of lead poisoning by Sir George Baker was a great medical advance. "Endemial colic" was of frequent occurrence in all parts of the world-in Spain, in France (Poitou), in Devonshire (where it had the name of "Devonshire colic"), and in Central America (where its local name was "dry belly-ache"). All these diseases, as a consequence of Baker's discovery were found to be due to the presence of lead in the blood and his paper, read at the Royal College of Physicians in 1767, was a masterpiece of reasoning and observation; Baker was assisted by Dr. Saunders, a chemist of great repute, in the chemical part of his investigations.

The fight with small-pox was another event which marked the medical progress of the eighteenth century; small-pox was then a veritable scourge. In 1717 Lady Mary Wortley Montagu, who accompanied her husband to Adrianople, relates that the Turks mitigated the attacks by the process of inoculation; she had her son inoculated. The practice was tried on some criminals in Newgate who were promised that they should go scot free if they recovered, which fortunately for themselves they did. After this it received the sanction of the profession in this country and inoculation became general, but experience proved that while the mortality from the inoculated disease was low the practice of inoculation favoured the spread of the disease.

In the latter half of the eighteenth century Edward Jenner, who was apprenticed at Sodbury in Gloucestershire, was struck by the answer of a woman who said that she could not have small-pox because she had had cow-pox; he never forgot this and made every inquiry possible relating to this belief, and after working for over 20 years disclosed his views on the subject. He performed the first vaccination of a boy in 1796. Jenner made a discovery of which we do not yet know the full meaning and which will be found to have a more general application than the discoverer himself could have thought of at the time. Many people made a great deal of money out of small-pox, amongst whom was Thomas Dimsdale, who published "The Present Method of Inoculating for the Small-pox," which ran through seven editions in three years. The Empress Catharine of Russia sent for Dimsdale to inoculate her and some of her family for which he received the very moderate fee of $£ 12,000$ and $£ 500$ per annum and was made a Baron; small-pox then was in some respects a very useful thing! Coming to the surgeons, Dr. Poore mentioned Cheselden, a great anatomist, who wrote a work on the bones, Percival Pott, and John Hunter. Hunter was one of the greatest geniuses who ever lived and a born naturalist. One had only to read the Lives of Hunter and
Harvey to see that their minds were hardly ever detas $3 x$ a d from the object of their lives. Hunter cared fow now except the dissection of animals and the colleation morbid specimens, and these characteristics accous the great amount of knowledge which be accummotad. Dr. Poore advised all present to read Mr. Stephen "s "Life of John Hunter," which was a delightfos 1:2) book. It brought to one's own mind John Husts in most remarkable way. A lady, in complimentis Paget on the book, said of Hunter: "But what a he was!" Some points in Hunter's character ons

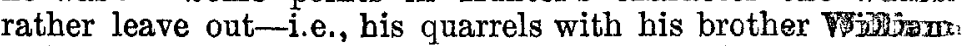
and other colleagues. It must also not be forgottes Jenner was his pupil.and Jenner held Hunter in the Josy highest admiration and always alluded to him as " thas man." It is not too much to say that Jenner had a gerome love for Hunter, and this feeling evinced by one of eminence for another stands greatly to the credit of $F_{\text {soxiter. }}$ Medicine has been the benefactor of many other sciezars. and amongst the medical men who have bsen prost scientists Dr. Poore mentioned Dr. Gilbert, physicies $7 D^{\circ}$ Queen Elizabeth, the parent of magnetism : Woodx parent in this country of geology; Grew and Fotherrom, Sloane and W. Hunter, great botanical collectors; Sasus res: and Wollaston, great chemists; Sir William Watson, the trician; James Young, propounder of the undelatery of light; and Wells, the physicist who distinguish mimself by his researches on dew. Dr. Poore concluded his-a by referring to the literary medical men of the eightssaits century, who were a remarkable body. Oliver Goldmmitisy of course, came first as one of the greatest writers of troptist this country had ever produced. Garth wrote satixical which were thought very much of at the time but if 1928 . 3 wn would appear dull work because the local colouring ald gone. John Arbuthnot, a mathematician and physician, $\$ 20$ a friend of Dr. Johnson, of Pope, and of Swift, on whose vretion. os it was difficult to say accurately what influence he bads. The: was probably about the only man Swift admired. Artsidnerst was said to have inspired a great deal of "Gullis: $\mathrm{T}^{2} \mathrm{~s}$ Travels" and was also concerned with Pope and Swift The production of the "Memoirs of Martinus Scriblerus," Drik of wit but somewhat coarse. Smollett, a great wit great novelist, and Dr. John Aikin, brother of Mrs. Baxisuld whose "Evenings at Home" had been translated into $\$ 3 y$ European language, closed the list. "In conclusion, gهollemen," said Dr. Poore, "I repeat what I said s's the beginning, that fame is very short-lived and that those admired to-day will probably be forgotten 50 y ars: hence. I began with Tennyson; I will end with Dr. Wists:-

$$
\begin{gathered}
\text { "Time, like an ever-rolling stream, } \\
\text { Bears all its sons away; } \\
\text { They fly forgotten as a dream } \\
\text { Dies at the opening day.", }
\end{gathered}
$$

\section{ON "CROSSED" APHASTA}

\section{AND THE FACTORS WHIOH GO TO DETETHISTS}

\section{WHETHER THE "LEADING" OR "DRTVIM "} SPEEOH-OENTRES SHALL BE LOCATED I

THE LEFT OR IN THE RIGHT HEMI SPHERE OF THE BRAIN,

WITH NOTES OF A CASE OF "CROSSED" APHASIA (AREGESJ WITH RIGHT-SIDED HEMIPLEGIA) IN A LEEIr HANDED MAN.

By BYROM BRAMWELL, M.D., F.R.C.P., F.R.\$. Figw,

PHYSICIAN TO THE ROYAI INFIRMARY, EDINBURGH ; LECIUREP OIS JTI PRINCIPLES AND PRACTICE OF MRIDICINE AND ON CLINICLS MEOICINE IN THE SCHOOL OF MEDICINE OF THE ROYAT. COLLFGES, RDINBURGH, ETC.

IN perfectly healthy right-handed persons who w inherit a tendency to left-handedness ${ }^{1}$ the "driwims" "leading" speech-centres are (with perhaps rare excemsixoms. but I know of no recorded cases which definitely prore this situated in the left hemisphere of the brain ; and vies in left-handed persons the "leading" or "driving" spermet centres are, so far as we know, usually, but probaldy 3 rse constantly, situated in the right hemisphere. Consequezridy.

1 My reasons for introducing the qualifications " perfectly weatwy apparent after the reader has perused the paper. 
when aphasia (I refer to marked and persistent and not merely temporary aphasia) occurs in a left-handed person the lesion is usually situated in the right hemisphere of the brain and the hemiplegia (if there is hemiplegia) is usually. left-sided. But in some cases, which so far as we at present know are quite exceptional, an attack of right-sided hemiplegia in a left-handed person is attended with aphasia -in other words, in exceptional cases the "leading" or "driving" speech-centres are in left-handed persons, situated in the left hemisphere of the brain. The following is an illustration in point.

A man, aged 36 years, for the past year a barman, formerly a pitman, was admitted to the Edinburgh Royal Infirmary on March 9th, 1899, suffering from right-sided hemiplegia and aphasia. The patient was a left-handed man and had been so all his life. He did everything except write with his left hand; he had always written with the right hand, but he could write a little with the left. None of his near relatives or ancestors were known to have been left-handed. He was a twin; his twin-brother was right-handed. The history as to left-handedness both in the patient and his relatives and ancestors had such an important bearing on the case that I both wrote to and interviewed the patient's mother on the subject. She told me that the patient was strongly left-handed from early infancy; in order to teach him to write with his right hand the schoolmaster strapped his left hand to his side; he had never written with his left hand; none of his relatives or ancestors either on the father's or mother's side had so far as was known been left-handed. With regard to his previous history (which was partly obtained from his wife and partly from himself) he never had any illness, so far as he remembers or his wife knows, until the present attack. He had not had syphilis. He had been a steady man. He knew no cause for the present attack. On Nov. 6th, 1898, while straining at stool he was suddenly seized with right sided hemiplegia and apbasia His wife said that when she went to the water-closet to see why he was remaining so long (10 minutes after he had gone to stool) she found him paralysed on the right side, unable to speak, but not unconscious. Up to the onset of the attack he was in the enjoyment of perfect health and had never suffered from headache, giddiness, vomiting, or any other symptoms suggestive of cerebral disease. For six weeks after the onset he was confined to bed. Dhing this time he appeared to be in a "dazed condition" and slept a great deal, but his wife stated that from se first he seemed to understand what was said to him and to know what he wanted to say. She also thought that after the first six weeks he was able to read, for he not only busied himself with the newspaper every day but on several occasions he directed her attention to paragraphs in the paper which had for him and her a special interest. At the end of six weeks his arm still remained completely paralysed but the right-sided facial paralysis had almost disappeared. He was beginning to move his leg in bed and he could say a few words. Since this date (the end of December, 1898) he has slowly but gradually improved. The family history of the patient was unimportant. He had been married for eight years and had had two children; the first was born at the full time, was alive and well and had always been healthy; the second was born dead at the full time. His wife had not had any miscarriages. The patient's present condition is as follows. $\mathrm{He}$ is a well-nourished, healthy-looking man. His height is five feet seven and a half inches and his weight is $12 \mathrm{st} .11 \mathrm{~b}$. He looks bright and intelligent. The temperature is normal. There is marked right-sided hemiplegia, the face, arm, and leg all being affected. The paralysis of the face is slight, though still detectable on careful examination. The paralysis of the leg is very marked; he is unable to stand or walk when unassisted, but he can do so if he has the support of an arm on his right side and a stick in his left hand. The paralysis of the arm is still more marked than that of the leg; it is complete in the hand. The right arm is markedly thinner than the left. The tendon reflexes are all exaggerated on the right side (ankle-clonus is present; the knee-jerk, the wrist-jerk, the triceps-jerk, and the jaw-jerk are all markedly exaggerated). On the left side there is no ankle-clonus and the left knee-jerk is normal in degree. The plantar reflex is marked on both sides. On tickling the sole the big toe of the right foot is extended-Babinski's reaction-while the toes of the left foot are flexed. The skin-sensibility to tactile and painful impressions is diminished in the right arm and leg; it is normal on the right side of the face. The sensibility to heat and cold seems equal and normal on the two sides of the body. The special senses are normal. There is no hemianopsia. The optic discs are normal. The heart, aorta, radial arteries, kidneys, and, in fact, all the internal viscera are perfectly normal. With regard to the speech functions, there is very marked motor-vocal aphasia, some word-deafness, considerable word-blindness and agraphia. The condition of the different speech faculties was in more detail as follows. As to the auditory speech-centre the patient seems to understand simple questions which are put to him and usually does simple acts which he is told to do correctly (shuts his eyes, puts out his tongue, holds up his hands, \&c.) but be occasionally makes mistakes when told to do such simple things (he once put out his tongue when told to close his eyes, for example) and evidently has considerable difficulty in understanding complicated spoken speech. Consequently there is some word-deafness. As to the motorrocal speech-centre he is almost completely unable to express himself in spoken speech. He can say his own name, "yes," "no," and a few other simple words, especially numbers; he seems to know what he wants to say, but cannot say it. He cannot, for example, tell me his wife's name or his child's name; he is annoyed at this. During the first three weeks that he was in hospital he never once correctly told me his age, but he knows quite well what his age is (36 years). When, however, he is asked to write his age with his left hand he always does so correctly in figures (36). He is able to indicate correctly (by holding up his fingers) the number of syllables in most words, though he makes some mistakes. On one occasion he indicated that there were two syllables in the words " $d$ gg" and "God," but on the same occasion he correctly indicated the number of syllables in the words " papa," "Constantinople," "Sunday," and "hippopotamus." He is unable to repeat the great majority of words which he is asked to say. He often repeats the same word over several times; but there are no special " recurring utterances." Consequently, there is marked motor-vocal aphasia. As to the visual speechcentre, he responds imperfectly to written questions even of a simple kind ("Put out your tongue," "Shut your eyes," "Give me your hand," \&c.). It was some time before he could be made to understand that these written questions were requests or commands to do something; and after he was made to understand this he did not always respond correctly in pantomime; for example, in answer to the written request, "Give me your hand," he put his hand straight up in the air ; he made out the word "hand" correctly but not the "give me." He can read figures much better than letters and words. He indicates at once, and correctly, by holding up his fingers the written numbers $4,6,9$, and 7 . He can add up a series of simple numbers, but takes time to do so, and often makes mistakes; for example, he made 10, 6, 7, and 5 when placed as an addition sum $=24$; when told that it was wrong he after a time made it 28 . He does not appear to be able to make out complicated written questions. Prior to his illness he was in the habit of reading the newspaper regularly. Consequently, there is a considerable degree of word-blindness. With regard to the motor writingcentre, he is unable to hold a pencil in his right hand (with which he was taught to write and with which he has only been in the habit of writing). He can write (i.e., express himself in writing), though very imperfectly, with his left hand. Prior to his illness, though he could write quite well he was not in the habit of writing much and he never wrote with his left hand. When asked to write his name, his age (36 years which he cannot utter), and some simple words and numbers such as "dog," $4,36,100$, he does so correctly with his left hand. But he is unable to write down other simple words; for example, he wrote " $c 0$ " when asked to write "cat," "gog" when asked to write "God" (he had immediately before this written the word " dog" : the " og" seemed to be running in his mind); when asked to write "one thousand and one" in figures he wrote 1000 and then 100 underneath it; when asked to write " ninety-eight" he wrote 92 , \&c. He writes with his left hand in the ordinary direction, from left to right (not in mirror writing). His inability to write is not due to any difficulty in forming the letters i.e., it is not a mere motor or kinæsthetic defect-but it appears to be due to loss of or want of the knowledge of the manner in which the letters should be grouped 
to form words-i.e., it appears to be, chiefly at all events, the result of an apbasic defect on the sensory side of the speech mechanism. He cannot, for example, spell such simple words as "cat" and "God" (in writing). Consequently there is marked agraphia; but it is diffecult to be sure of the extent of the agraphia since the patient has not been accustomed to write with the left hand.

Remarks.-The case presents several points of interest. The most important and the only one which I shall refer to at any length, is the fact that in this patient, who for everything except writing is left-handed, an attack of right-sided hemiplegia has been attended with marked and persisting aphasia ("crossed" aphasia). But before considering this point it will be necessary to discuss the diagnosis.

Anatomical diagnosis.-There can, I suppose, be little or no doubt (because of the presence of associated right-sided hemiplegia) that the lesion is situated in the left hemisphere of the brain. The only other possible explanation is that the lesion is situated in the right hemisphere (on the side of the hemiplegia) and that the pyramidal tracts do not decussate in the normal way at the top of the spinal cord. But the presence of such an anatomical variation is most improbable, consequently this explanation is infinitely less likely than the one which supposes that in this 'left-handed man the "leading" speech-centres are situated in the left hemisphere of the brain. I conclude, therefore, that the lesion is in the left hemisphere and that the aphasia is "crossed," in other words, that in this left-handed man the "leading" or "driving" speech-centres are not situated, as they usually are-so far as our present information enables us to judge-in left-handed persons, in the right, but that they are situated in the left hemisphere of the brain. The aphasia is partly motor and partly sensory (there is marked motor-vocal aphasia, some word-deafness, considerable word-blindness, and agraphia), but the motor-rocal aphasia appears to be the chief aphasic defect, and the statement of the patient's wife would seem to show that this has been the case since the early stages of the attack. The fact that there is not only motor-vocal aphasia but some word-deafness and considerable word-blindness proves, I think, conclusively that the lesion is cortical and that it involves the "zone of language"-the motor-vocal speech-centre (Broca's convolution) - and probably also to some extent, and perhaps to a considerable extent, the other speech-centres-auditory and visual-for it must be remembered that five months have elapsed since the occurrence of the lesion and that during this period considerable improvement in the sensory speech defects may have taken place. There is no reason whatever to suppose that the patient's inability to understand complicated spoken speech and written speech is due to a mere defect of intelligence it is obviously a speech defect-in other words, it is due to word-deafness and word-blindness. Nevertheless, although the aphasia is cortical the patient does respond-though not quite perfectly - to the Proust-Lichtheim test, for he can correctly indicate the number of syllables in such a complicated word as "Constantinople." Although in many cases of aphasia it is hazardous to predict the exact position of the lesion from the clinical phenomena alone, in this particular case it may, I think, for the reasons advanced above, be concluded: (1) that the lesion is situated in the left hemisphere; (2) that the motor side of the speech mechanism is directly implicated (though the sensory speech-centres are probably also involved); and (3) that the lesion is cortical.

Pathological diagnosis.-This does not call for any lengthened discussion. The sudden occurrence of the lesion while straining at stool shows that the lesion was a vascular one. Thrombosis may be excluded, because of the sudden onset and the absence of any previous symptoms indicative of cerebral disturbance or of vascular disease. The lesion was therefore either an embolism of the middle cerebral artery or a cerebral hæmorrhage-but which of these it is difficult or impossible to say. The patient is only 36 years of age; there is no kidney disease and no vascular disease; his wife said that he was not unconscious when she found him 10 minules after the onset. These facts are opposed to an intra-cerebral hæmorrhage and so is the cortical position of the lesion. On the other hand, the sudden occurrence while straining at stool is in favour of hæmorrhage, and especially of an extra-cerebral hæmorrhage (but it is most unlikely that a meningeal hæmorrhage would have produced such marked and persistent paralysis and aphasia), and is against embolism, and so is the absence of any cardiac or aortic lesion-i.e., any obvious cause of embolism. I conclude, then, that the lesion was either embolism or hæmorrhage and that the balance of evidence is, on the whole, in favour of the former (embolism).

The explanation of "crossed" aphasia.-And now I come to the point of chief interest in the case--viz., the "crossed" aphasia. The patient is a left-handed man, though he has always written, and only written, with his right hand; for everything else except writing he is left-handed. None of his near relatives or ancestors have been left-handed. He was suddenly seized with right-sided hemiplegia and aphasia the result of a vascular lesion in the left hemisphere. The aphasia is chiefly motor-vocal aphasia (though there is also considerable sensory aphasia) and the lesion is undoubtedly cortical. The severity of the motor-vocal aphasia and its long duration-four months -in a marked degree seem to prove that the destruction of Broca's convolution (the left motor-vocal speech-centre) must have been very complete. How, then, are we to account for the fact that in this left-handed man the "leading" or "driving" speech-centres are situated in the left and not in the right hemisphere of the brain? I will, for the sake of clearness and simplicity of argument, limit my remarks to the motorvocal speech-centre, leaving out of account the sensory aphasia which was also present.

In discussing this question of "crossed" aphasia it is essential to remember: 1 . That the vast majority of mankind ase one hand more than the other-i.e., are either right-handed or left-handed. Very few persons are actually ambidextrous; indeed, it is doubtful if any person is ever literally ambidextrous-i.e., able to use both hands equally well for every movement and action. Almost every personprobably every person-who at first sight appears to be ambidextrous will on careful examination be found to use one hand in preference to the other in the performance of some movements-i.e., the movements which for him are the most difficult and most unaccustomed. Further, it will generally be found that persons who appear to be ambidextrous are, or were, originally left-handed and that their ambidexterity is due to the fact that they have by practice and use trained the right hand to great dexterity notwithstanding the circumstance that they are, in consequence of special organic aptitude, left-handed. If right-handed persons-even those who are strongly right-handed from birth-were trained to use the left hand (in the same way that left-handed persons are trained to use the ric 'it hand) ambidexterity could, of course, be equally produced in them and would be frequently seen in right-handed persont but, as a matter of fact, they are not so trained, con sequently, right-handed persons are very rarely markedly ambidextrous. 2. That the great majority of persons are right-handed. Right-handedness is the rule, lefthandedness is the exception, and the exceptions-lefthanded people-constitute a minority (according to Hyrtl 2 per cent.) of the whole. Various theories have been advanced to account for this. I do not propose to discuss them in this paper further than to say that, however right-handedness may in the first instance have originated, $(a)$ according to some authorities it is the result of acquired habit; and (b) according to others it is the result of hereditary infiuence or special organic aptitude; and that both of the causes may without doubt be effective. Sir Daniel Wilson, after an exhaustive consideration of the whole subject, comes to the conclusion: "That the preferential use of the right hand is natural and instinctive with some persons ; that with a smaller number an equally strong impulse is felt prompting to the use of the left hand; but that with the great majority right-handedness is largely the result of education." 2 3. That the ancestors of the great majority of persons have for generations been righthanded. There is strong evidence to show that from the earliest times of which we have any accurate records on the point right-handedness was the rule and left-handedness was the exception. On this point Sir Daniel Wilson states: "So far then it seems to be $\mathrm{pr}_{3}$ ved that not only among cultured and civilised races, but among the barbarous tribes of both hemispheres-in Australia, Polynesia, among the Arctic tribes of our northern hemisphere at the present day, and among the palæolithic men of Europe's post-pliocene times-not only has a habitual preference been manifested for the use of one hand rather than the other but among all alike the same hand has been preferred. Yet, also, it is no less noteworthy that this 
prevailing uniformity of practice has always been accompanied by some very pronounced exceptions. Not only are cases of exceptional facility in the use of both hands of frequent occurrence, but while right-handedness everywhere predominates left-handedness is nowhere unknown." 3 4. Consequently, (a) heredity, (b) congenital organic aptitude, and (o) actual acquirement (habit, example, social usage, and education) all tend to make the great majority of persons right-handed. It must also be remembered that left-handedness is sometimes hereditary and may run through several generations. In many-probably most-cases, however, in which a person is left-handed the left-handedness, though congenital-i.e., born with the individual and the result of what may be termed organic aptitude-is not, strictly speaking, hereditary. Why some persons whose ancestors were not left-handed should be born witk a strong tendency to use the left hand rather than the right we do not know any more than we know why in some (rare) cases the viscera are transposed. Again, the descendants of left-handed ancestors may be right-handed even when the left-handedness has been present in several preceding generations. I take it that in such cases there is (it may be even after several generations of left-handedness) a reversion to the original right-handed condition. 5. It consequently follows that in right-handed persons the left hemisphere and in left-handed persons the right hemisphere is the "leading" or "driving" side (hemisphere) of the brain. I need not discuss the question whether the greater activity or superiority, if the term may ke allowed, of the left hemisphere in right-handed persons and of the right hemisphere in left-handed persons is the cause or the consequence of the right or left-handedness. 6. That with rare exceptions the "leading" or "driving" speech-centres are situated in the hemisphere of the brain which is, so far as the movements of the hand are concerned, the "leading" or "driving" side. This is a fundamental proposition founded on the results of clinical and pathological observation which is acknowledged by all authorities. Consequently, in right-handed persons the "leading" or "driving" speech-centres are, with perhaps rare exceptions, situated in the left hemisphere; and in the great majority of left-handed persons the "leading" or "driving" speech-centres are situated in the right hemisphere, and therefore (7) acute and complete destruction of Broca's convolution (the left motor-vocal speech-centre), when it occurs in a right-handed man, will, and does, in the vast majority of cases produce motor-vocal aphasia; while the same lesion when it occurs in a left-handed man will not, and does not, in most cases produce motor-vocal aphasia. Vice vers $\hat{a}$, acute and complete destruction of the motorrocal speech-centre in the right hemisphere of the brain when it occurs in a left-handed man will, and does, in most cases prcduce motor-vocal aphasia; while the same lesion when it occurs in a right-handed man will not, and does not, in the vast majority of cases produce motor-vocal aphasia. I mean, of course, marked and persisting, and not merely temporary, motor-vocal aphasia. ${ }^{4}$

Exceptions to these statements occasionally occur. I term such exceptions cases of "crossed" aphasia. In the vast majority of cases of "crossed" aphasia the speech defect is merely temporary and evanescent. It is by no means uncommon for a certain degree of motor-vocal aphasiaobviously the result not merely of an intellectual defect but of a speech defect-to attend the onset of an attack of left-sided hemiplegia in a right-handed person, and probably also to attend the onset of an attack of right-sided hemiplegia in a left-handed person (though from the comparative paucity of left-handed people the opportunity of observing such cases rarely occurs). Cases of temporary "crossed" aphasia are, I think, easily enough explained if it be granted, as I think it must be granted-and the occurrence of these cases of temporary "crossed" aphasia is one argument in favour of this view-that the cortical centres in the "non-leading" or "non-driving" hemisphere of the braini.e., the right hemisphere in right-handed persons and the left hemisphere in left-handed persons-which correspond to the speech-centres in the "leading" or " driving" hemispherei.e., the left hemisphere in right-handed persons and the right hemisphere in left-handed persons-are possessed of some sort or degree of speech function, which is usually, I

$$
3 \text { Ibic., p. } 115 .
$$

4 For the sake of simplicity I limit my remarks to motor-vocal aphasia, but the same statement may be applied, though not perhaps curite so exactly, to the sensory varieties of aphasia. think, carried on in conjunction with, and in subordination to, the function of the speech-centres in the "leading" or "driving" hemisphere and which probably, I think, varies in degree in different individuals and in accordance with the function of the individual speech-centres (auditory, motorrocal, and visual). Cases of "crossed" aphasia are, however, occasionally, though very rarely, met with in which the aphasic symptoms are of long duration-i.e., are persistent or permanent. But so far as I know cases of this kind very rarely indeed accur in right-handed persons, indeed I know of no recorded case; so far as I know they almost always occur in left-handed persons. I am speaking of cases in which there is no reason to suppose that the normal development of the speech-centres was interfered with in early life.

The factors which go to determine whether the "leading" or " driving" speech-centres shall be located in the left or in the right hemisphere of the brain seem to me to be chiefly two-viz.: (1) heredity; and (2) congenital aptitude or personal acquirement-i.e., whether the person is right-handed or left-handed; and, speaking generally, I am disposed to think that the latter would, if these were the only factors, be, in most cases at all events, the more potent of the two. For example, in the majority of left-handed persons whose ancestors were right-handed-i.e., who inherit no tendency to left-handedness-the influence of the left-handedness, which tends to locate the "leading" or "driving" speechcentres in the right hemisphere, which because of the lefthandedness is in them the "leading" hemisphere so far as the movements of the hand are concerned, will be more potent than the influence of the heredity, which tends to locate the "leading" or "driving" speech-centres in the left hemisphere, which in preceding generations was the "leading" hemisphere both as regards the movements of the hand and also as regards the speech-centres. Hence, if heredity and personal acquirement were the only factors the "leading" or "driving" speech-centres would in the majority of left-handed persons be situated in the right hemisphere. Whether the act of learning to write with the right or the left hand and the practice of the act of writing after the act of writing has been acquired have any influence in locating the "leading" or "driving" speech centres in one or other-the left or the right-hemisphere of the brain is an interesting question and one which it is perhaps impossible to answer with any degree of certainty. We learn to read and write long after we learn to understand spoken language and to speak, and long after we have become (whether this is the result of heredity, of congenital peculiarity, or of acquired habit) right-handed or left-handed. The fundamental speech-centres, as I term them, or the "primary couple," as Wyllie has termed them, are trained and their functional activity is highly developed long before the accessory speech-centres (the secondary couple) are trained and brought into action. It may therefore, I think, reasonably be supposed that in most persons the location of the fundamental speech-centres (auditory and motorrocai), as the "leading" speech-centres in the left hemisphere, has already been fixed and determined before the associated speech-centres (the visual speech-centre and the motor-writing centre) begin to be called into action. In right-handed persons, in whom the location of the fundamental speech-centres, as "leading" speech-centres, in the left hemisphere has already been determined both by heredity and actual acquirement, the fact that they are taught to write with the right hand will confirm and strengthen this location. But whether in left-handed persons whose ancestors were right-handed-in whom the fundamental speech-centres have been already located as the "leading" centres, in the right hemisphere, by the preponderating influence of actual acquirement or congenital peculiarity over heredity-the fact that they are taught to write with the right hand can upset this (prior) localisation -i.e., can re-transfer the "leading" speech-centres to the right hemisphere-is an interesting question. It is probable, I think, that in some cases it may be able to do so.

It seems to me that in left-handed persons whose ancestors have been right-handed the question whether the "leading" or "driving" speech-centres are located in the right or in the left hemisphere of the brain will-at all events, prior to the time when they are trained to write with the right hand-chiefly depend upon the fact whether the hereditary influence which favours their location in the left hemisphere or the acquired influence-due to the lefthandedness-which favours their location in the right hemisphere, is the more potent, and, as I have already stated, 
I am disposed to think that, in most cases at all events, the latter factor will be the more powerful. But it is not difficult to conceive that in some cases (left-handed persons whose aucestors were right-handed) these two factors-heredity and actual acquirement-will, so far as the location of the speech-centres is concerned, be pretty evenly balanced, and that up to the time that the child begins to learn to read and write the location of the speech-centres as the "leading" speech-centres in the right hemisphere (even granting that the influence of the acquired left-handedness is more potent than the influence of heredity) will not be very firmly fixed. And in such cases it is not, I think, unreasonable to conclude that the introduction of a third factor-viz., the learning to write with the right hand, the most highly specialised movement, it must be remembered, of the hand so far as the speech functions are concerned-may be sufficient to turn the scale and to determine that the "driving" or "leading" speechcentres (the location of which up to this point is not very firmly fixed in the right hemisphere) shall be, finally and firmly, located in the left hemisphere.

Hence in cases of "crossed" aphasia in left-handed persons, with the object of trying to throw further light upon this point, it should always be noted whether the patient has been taught to write with the right or the left hand and whether he has been accustomed to write much or little and whether he is ambidextrous or not. In the particular instance I have recorded the patient was taught to write with his right hand and had never learned to write or been in the habit of writing with his left hand; hence the third factor, which I have suggested as a possible cause of the location of the "leading" speech-centres in the left hemisphere of the brain in left-handed persons, may perhaps in this particular case have come into play and may have finally determined the location of the "leading" speechcentres in the left hemisphere. And in this connexion it is interesting to note that Dickinson's case of "crossed" aphasia in a left-handed man (recorded by Bastian) is in many respects very similar to my own-viz.: right hemiplegia with marked motor aphasia in a left-handed man who was so ambidextrous that although " he was in common phrase left-handed he said he was not to be called so since he used both hands equally well." o Further, this view (the theory that the act of learning to write and the practice of the art of writing with the right hand may, in some lefthanded persons, determine the location of the "leading" or "driving" speech-centres in the left hemisphere of the brain) seems to be supported by the following case recorded by H. Oppenheim.

A woman, aged 59 years, who had been left-handed since the age of 17 years in consequence of an injury received at that time to the right hand, was admitted to hospital on Sept. 23rd, 1889, and died on 'Oct. 6th, 1889. For two years before admission to hospital she had suffered from headache. 14 days before admission to hospital aphasic symptoms (complete word-blindness and agraphia, some word-deafness, and paraphasia) gradually developed. During her stay in hospital she became completely hemiplegic and hemi anæsthetic on the left side; left-sided bilateral homonymous hemianopsia was found to be present; and optic neuritis developed in the right eye three days before death. On postmortem examination a large sarcomatous tumour was found in the right hemisphere. It involved the optic thalamus, the lenticular nucleus, the internal capsule, and extended up to the island of Reil and into the white matter of the occipital lobe. There was no lesion, either macroscopic or microcopic, in the left hemisphere.

This case would seem to show that, even at the age of 17 years the "leading" or "driving" speech-centres, in a right-handed person, may-I do not, of course, say that they will always-or will usually, if the patient becomes lefthanded in consequence of disuse of the right hand, be transferred from the left to the right hemisphere. At all events this seems to me to be the most reasonable explanation of the facts in this very important and most remarkable case, and it is the explanation which Oppenheim himself suggests. ${ }^{7}$ And if this explanation is accepted it certainly lends support to the "third factor" supposition advanced

5 Aphasia and Other Speech Defects, p. $\complement_{4}$.

Archiv für Psychiatrie, vol. xxi., 1890, p. 13

7 The only other explanation would be that the "

"driving" speech-centres were in this previously right-har

originally situated in the right hemisphere, but this explans much less likely and much more difficult to accept. above. I am disposed, therefore, to think that the exceptional cases in which in left-handed persons the "leading" or "driving" speech-centres are situated not as one would expect in the right but in the left hemisphere of the brain may perhaps in most cases be best explained by supposing that in them the influence either of $(a)$ heredity or $(b)$ of heredity + the third factor which I have suggestedviz., the learning to write and the practice of the art of writing with the right hand-was, so far as the localisation of the "leading" or "driving" speechcentres is concerned, stronger than the influence of the acquired (non-hereditary) left-handedness; that is to say, in such exceptional cases the hereditary localisation of the "leading" or "driving" speech-centres in the left hemisphere (or hereditary + the third factor) have prevented the transference of these (the "leading" or "driving") speech-centres to the right hemisphere. If this supposition is correct one would consequently expect that in most of the exceptional cases in which a left-handed person becomes aphasic and hemiplegic on the right side as the result of a lesion in the left hemisphere of the brain the ancestors of the patient will have been right-handed (i.e. the left-handedness will be acquired and not hereditary), and probably also the patient will have been taught to write and accustomed to write with the right hand, or at all events will be ambidextrous-i.e., accustomed to use the right hand almost as well as the left. And so far as I know this is usually the case. So far as I know no case has been recorded in which "crossed" aphasia was developed in a left-handed person whose ancestors had for several generations been left-handed, though it is probable, I think, for the reason given below, that such cases may occasionally, though probably very rarely, occur. Nor, so far as I know, has any case of "crossed" aphasia in a left-handed person been recorded in which the patient had been taught to write and had only been in the habit of writing with the left hand. Vice versâa one would expect that in cases in which "crossed" aphasia of a marked and persistent kind occurred in a right-handed person some of the near relations or ancestors of that person would probably have been lefthanded ; in other words, that the development of "crossed" aphasia in right-handed persons would be due to the fact that the influence of heredity in keeping the "leading" speech-centres located in the right hemisphere-i.e., the hemisphere which in some of the patient's near relations or ancestors was the "leading" hemisphere-was stronger than the influence of the acquired right-handedness in transferring the "leading" speech-centres to the left hemisphere. But in cases in which some of the near relations or ancestors of a right-handed person had been left-handed the influence of heredity in keeping the "leading" speechcentres located in the right hemisphere would probably be less potent and constant than the influence of heredity in keeping the "leading" speech-centres in the left hemisphere in the case of a left-handed person whose ancestors had been right-handed in consequence of $(a)$ the tendency which there is in all abnormal organisms to ultimately revert to the original (normal) condition-i.e., to right-handedness and left-brainedness in the special case which we are considering $; 8$ and $(b)$ that in both cases (since left-handed persons as well as right-handed persons are usually taught to write with the right hand) the "third factor" will tend to locate the "leading" speech-centres in the left hemisphere. So far as I know, "crossed" aphasia of a marked and persisting kind in a previously healthy ${ }^{9}$ right-handed

Though right-handedness is not perhaps, strictly speaking; the original condition, it (right-handedness) may, I think, so far as the original condition, it (right-handedness) may, I think, so far as the speech-centres are concerned, for practical purposes be regarded as the original condition. Whether man was right-hanced and therefore left-brained before the speech functions and speech faculties became
highly differentfated and developed we do not know. But, looking at the long periods of time during which the majority of men have been right-handed and left-brained, left-brainedness may be considered, so far as the localisation of the "leading" speech-centres is concerned, as the usual (normal) and original condition. By the term "leftbrained "I merely, of course, mean that the left hemisphere of the brain is the "leading" or "driving" hemisphere.

9 By a previously heaithy right-handed person I mean a righthanded person in whom there was no cerebral lesion in early life tu interfere with the normal (usual) development of the speechcentres. It is obvious that if in a right-handed child Broca's convolu. tion were destroyed or arrested in its development, the motor-vocal speech-centre in the opposite (right) hemisphere would become tho "leading" motor-vocal speech-centre; and consequently, that if in ater life such a person were ta become hemiplegic in consequence of a esion in the right hemisphere, the left-sided hemiplegia in such right-handed person would be associated with aphasia-ie the aphasia would be "crossed." Further, it may perhaps be supposed 
person and due to a lesion in the right hemisphere is an extremely rare condition (indeed, $\mathrm{r}$ know of no such cases and I will be very much obliged to any of my readers who can refer me to any) ; but "crossed" aphasia in left-handed persons, though also very rare, seems to be more common, more especially when it is remembered that according to Hyrtyl 98 per cent. of civilised mankind are right-handed and only 2 per cent. left-handed. The rarity of "crossed" aphasia in right-handed persons in comparison with "crossed" apbasia in left-handed persons seems to support the views advanced above.

In a recent issue of THE LANCET ${ }^{10}$ Dr. James $\mathrm{S}$. Collier recorded a very interesting and important case in which the left motor-vocal speech-centre (Broca's convolution) was completely destroyed in a right-handed man by a neoplasm and in which there was no aphasia. In his able and interesting comments on the case he suggests that the case proves that, "notwitbstanding the immense influence of heredity, right-handedness may in rare cases be associated with leading speech-centres in the right hemisphere." In support of this view he advances the fact that left-handedness does not always transfer the leading speech-centre to the right side of the brain, as exemplified in Dickinson's case, ${ }^{11}$ and $\mathbf{I}$ may add my own case recorded above. ${ }^{2}$ The same explanation was advanced by Dr. Ford Robertson at a meeting of the Edinburgh Pathological Club to account for the absence of aphasia in my case ${ }^{13}$ to which Dr. Collier refers in his paper. But the argument does not carry conviction to my mind.

The fact that in left-handed persons whose ancestors were not left-handed-i.e., who were right-handed-a leftsided lesion occasionally though rarely produces aphasia (because, I suggest, either the influence of heredity or the influence of heredity combined with the third factor-learning to write with the right hand-has in such exceptional cases been too strong to allow the "leading" speech-centres to be transferred by the acquired left-handedness to the right hemisphere) does not seem to me to warrant the conclusion that in right-handed persons whose ancestors were righthanded and who have been trained to write and who have been in the habit of writing only with the right hand the "leading" or "driving" speech-centres may be transferred (without, be it noted, any apparent reason or obvious cause, and in opposition both to the influence of heredity and of actual acquirement) to the right hemisphere.

The occurrence of "crossed" aphasia in a left-handed person whose ancestors were right-handed can, I think, in some cases at all events, be explained by the preponderating influence of heredity or by the combined influence of heredity and the act of learning to write with the right hand in keeping the "leading" or "driving" speech-centres located in the left hemisphere. The occurrence of "crossed" aphasia in a right-handed person whose ancestors were lefthanded, or who himself, as in Oppenheim's case, though originally right-handed had for some years been lefthanded can be explained in the same way-i.e., by heredity or by transference due to the acquired lefthandedness. The occurrence of "crossed" aphasia in a left-handed person some of whose ancestors were lefthanded (if such cases occur) can also, I think, be probably explained-viz., by reversion to the original condition-i.e., left-brainedness so far as the speech-centres are concerned. But the occurrence of "crossed" aphasia in a right-handed person whose ancestors were right-handed and who had been trained to write with his right hand cannot be explained in this or, so far as I see, in any other way;

that the child of a right-handed person whose motor-vocal speechcentre was in consequence of such a lesion in early life situated in the "ight hemisphere might inherit this location of the "leading" or "driving" motor-vocal speech-centre; in other words, that he (the child of such a person), though himself perfectly healthy and righthanded, might have as the result of hereditary transmission his "leading" or "driving" motor-vocal speech-centre situated in the right hemisphere of the brain. And consequently if he (such a righthanded person) became hemiplegic on the left side he would at the same time become aphasic. Though I conceive that such an explanation of "crossed" aphasia in a right-handed person is theoretically possible, the tendency to "revert" to the criginal left-brained condition) likely to occu in actual practice.

11 Bastian : Aphasia and other Speech Defects, 1898, p. 90.

12 Wadham's case, to which Dr. Collier refers, was not a case of "crussed" aphasia. In it a lesion in the right hemisphere prohuce left hemiplegi: and arhasia in a left-handed man who, though
originally lett-tanded, was amsidextrous and able to write, not only with 1 he lett, hut also witi the right hand (though his mother had, prior to the aphasic attack, never seen him write with bis right hand). Brain, 1898, p. 343 . in cases of this kind the influence of all three factors-viz., (a) heredity, (b) actual acquirement, and (c) learning to write and the act of writing with the right hand-would tend to keep the "leading" speech-centres located in the left hemisphere.

I do not, of course, presume to assert that the "leading" or "driving" speech-centres in a right-handed person whose ancestors have always been right-handed and who has himself been trained to write with the right hand cannot be (are never) located in the right hemisphere. I merely say that so far as I know the evidence which we at present possess is insufficient to support such a riew and that even granting that such a transference of the "leading" or "driving" speech-centres may and does occur $I$ see no satisfactory explanation to account for its occurrence. I am speaking, it must be remembered, of cases in which the patient was previously healthy-i.e., of cases in which there is no reason to suppose that there was any lesion in early life (either in the individual himself or in his immediate predecessorssee footnote 9 ) on the left side of the brain to interfere with the normal development of the speech-centres. Although I believe that in some exceptional cases the speech. centres in the right hemisphere are, in right-handed persons, sufficiently active and educated to immediately take up and independently carry on the speech functions when the "leading" or "driving" speech-centres in the left hemisphere are acutely and completely destroyed, I see no sufficient reasons for doubting that in such cases the speechcentres in the left hemisphere were, prior to the occurrence of the lesion, the "leading" or "driving" speech-centres. The fact that Broca's convolution may in a right-handed man be completely destroyed without the production of motor-vocal aphasia, as in the case which Dr. Collier records, ${ }^{14}$ and as in my case, to which he refers, ${ }^{15}$ does not, I maintain, prove that the "leading" or "driving" speechcentres were in such right-handed persons situated in the right hemisphere. Such cases seem to me merely to show that the motor-vocal speech-centre in the right hemisphere was, in such persons, capable of taking up and independently carrying on the speech functions when the opposite and, as I believe, the previously "leading" or "driving" motor-vocal speech-centre in the left hemisphere was destroyed. I take it for granted (for clinical and pathological evidence seems strongly to support this view) that the speech-centres in one or other hemisphere will always take the lead.

And here I must emphasise the fact that it is of the utmost importance to draw a distinction between the cases, such as my case to which Dr. Collier refers, in which the left motor-vocal speech-centre was suddenly destroyed, and cases, such as Dr. Oollier's, in which the motor speech-centre was slowly and gradually destroyed. The explanation which seems to me to afford the best solution of the one (the acute cases), does not, in my opinion (though it may possibly in rare cases be the correct explanation), afford the best solution of the other (the chronic cases). In those cases, such as the one I bave recorded, in which the destruction is sudden, the most reasonable explanation seems to me to be that the opposite (right, and, I believe, previously "non-leading") motor-vocal speech-centre was from the first-i.e., immediately after the occurrence of the lesion and without any process of training or substitution-sufficiently educated and active to take up and independently carry on the speech function when the normally "leading" or "driving" speechcentre in the left hemisphere was (acutely and completely) destroyed. This, for the sake of reference and convenience, I will term the "highly specialised" theory.18 In cases such as Dr. Collier's in which the destruction is slowly and gradually produced-i.e., by a neoplasm-the most reasonable explanation seems to me to be that

14 The LANCET, March 25 th, 1899 , p. 824 15 Brain, 1898 , p. 343.

18 It may, of course, be argued that if one "grants that in a righthanded person whose ancestors were right-handed the functional activity of the speech-centres in the right (non-leading) hemisphere may in rare and quite exceptional instances be so great as to enable them to take up and independently carry on the speech function more or less satisfactorily, but never, so far as I know, quite perfectly, immediately after the leading speech-centres in the left hemisphere are suddenly and acutely destroyed, why not go further and grant that they-the speech-centres in the right hemisphere of a right-hande man-may in rare and exceptional cases be the "leading" centres. admit that it is impossible to disprove this possibility, but I submit that analogy and the results of clinical and pathological experience seem to point the other way. 
pari passu with the destruction of the "leading" or "driving" motor-vocal speech-centre in the left hemisphere, the corresponding and hitherto "non-leading" motorvocal centre in the right hemisphere becomes educated and trained, so that even if the destruction is complete it is able to independently carry on the function of the "leading" speech-centre in the left hemisphere when that "leading" speech-centre is destroyed. This I term the "substitution or compensation" theory.

Substitution and compensation are probably, I think, more easily and more perfectly established in the case of slow and gradual than in the case of acute destruction of a speechcentre, granting of course that the time element (the period allowed for substitution and compensation) is the same in the two cases. What I mean to say is that supposing a tumour completely but slowly and gradually destroys the motor-vocal speech-centre in the course of six months the compensation (motor speech function) will at the end of that time be more perfect than in a case in which the motor rocal speech-centre was suddenly and completely destroyed and in which six months (the same time) has been allowed for the process of compensation and substitution to take place. My reasons are that in the case of acute destruction (1) the cerebral speech mechanism as a whole (the sensory speech-centres as well as the motor speech-centre) is usually, in some degree at all events, thrown out of gear ; and (2) irritative and inhibitory disturbances are apt to be produced in the sensory speechcentres, more especially if the lesion is, as it often is, at its commencement, irritative in character. Whereas, in the case of slow and gradual destruction of the motor-vocal speech-centre, the sensory speech-centres are not thrown out of gear and are not so apt to be disturbed by irritation or inhibition, since the lesion-usually a glioma or a gliosarcoma-is not usually irritative in character. Hence in the case of slow and gradual destruction of the left motorvocal speech-centre, the sensory speech-centres can, from the very commencement of the destruction and during the whole of the period which is allowed for substitution and compensation, play upon and educate the right motor-vocal speech-centre in a more natural and normal (undisturbed) manner. And if the destruction of the "leading" motorrocal speech-centre in the left hemisphere is slowly and gradually established I see no reason to suppose that any aphasic symptoms will necessarily be produced during the process of its destruction-i.e., during this period of substitution and training.

It may, of course, happen (though this is, I fancy, very rare) that before the tumour began to develop the motorvocal speech-centre in the right hemisphere was exceptionally active. In short, the non-occurrence of aphasia in the chronic cases (such as Dr. Collier's case) may perhaps in rare instances be due to the same reason which $\mathbf{I}$ have advanced to explain the absence of aphasia in the acute cases. But in the nature of things it would be impossible to prove thisi.e., to show that recovery was not the result of compensation and substitution. But this is, I think, a much less probable explanation than that which the "substitution or compensation" theory affords. We know that substitution and compensation do constantly occur. Hence in those cases in which there is sufficient time for the development of substitution and compensation it is only reasonable to accept the "substitution and compensation" theory as a sufficient explanation rather than to have recourse to the "highly specialised" theory, which is much more difficult to accept, but which notwithstanding the difficulty of accepting it seems to me to be the only reasonable explanation in those cases, in which the destruction is acute, since in the acute cases there is no time for substitution and compensation to occur. Dr. Collier's reason for'rejecting the substitution theory in the case which he has recorded is that "it is inconceivable that the glossokinæsthetic function could have been taken up by the right third frontal convolution pari passu with the devolution of the left third frontal convolution owing to the presence of a slowly growing neoplasm without some speech defect being at any time noticeable." With this opinion I cannot agree. If an ordinary motorcentre-the cortical centre for the hand, for examplecan be completely destroyed by a neoplasm without the production of any paralysis-in other words, if in the case of the ordinary motor-centres substitution and compensation can occur-surely they may also occur in the case of the notor-vocal speech-centre, the cortical representation of which is at least as bilateral-personally I am disposed to think more bilateral - than the cortical representation of the hand. Now, cases in which the motor-centre for the hand is completely destroyed without any paralysis being developed do undoubtedly occur. I bave myself met with two cases of this kind (Cases 1 and 2 following). The same thing also occurs in the case of the speech-centres. Dr. Collier's case is, I maintain, an excellent illustration in point, and it is not singular. I have met with two cases of the same kind (Cases 2 and 3 following); two cases in which in right-handed persons whose ancestors were not left-handed the left motor-vocal speech-centre was completely destroyed by a neoplasm and in which there was no motor-vocal aphasia.

These cases are reported in the last number of Brain (in my series of fatal cases of intracranial tumours). The headings of the cases are as follows :Case 1 (Case 7 in the series).-A large sarcoma springing from the dura, causing extensive atrophy and destruction of the right ascending frontal and ascending parietal convolutions, the inferior and middle frontal convolutions, the outer half of the island of Reil, and the anterior end of the temporo-sphenoidal lobe; no paralysis.-Case 2 (Case 9 in the series).--Large glioma and cyst in the left hemisphere, causing complete destruction of the greater part of the left ascending frontal and ascending parietal convolutions and their subjacent white matter, the posterior end of the third left frontal convolution, and the greater part of the white matter of the left frontal lobe. No motor paralysis till four days before death; no aphasia.-Case 3 (Case 10 in the series).-Enormous gliomatous tumour involving and destroying the greater part of the left frontal and temporosphenoidal lobes, and a large part of the left occipital lobe; no aphasia.

Dr. Ford Robertson has also given me the reference to another case of exactly the same kind-viz., complete destruction of the motor-vocal speech-centre in the left hemisphere by a tumour without the production of aphasia. The case is reported by Giulio Levi, who, in commenting on the case, refers to Oppenheim's case (abstracted above) and advances the same theory which Dr. Collier has advanced to account for the absence of aphasia. ${ }^{17}$

I maintain, then, that the non-occurrence of motor aphasia in cases in which Broca's convolution is slowly and gradually destroyed can be quite satisfactorily accounted for by the "substitution-compensation" theory, and that, so far as I know, no sufficient grounds have as yet been advanced to support the view that in right-handed persons whose ancestors were right-handed the "leading" or "driving" motor-vocal speech-centre may be situated in the right hemisphere of the brain. Though, as $I$ have already stated, I am not, of course, prepared to say that this cannot and does not occur.

Edinburgh

\section{THE RATIONAL TREATMENT OF CON- SUMPTION.}

Br J. G. SINCLAIR COGHILL, M.D., F.R.C.P. EdIN., M.R.C.P. LoND.,

SENIOR PHYSTCIAN TO THE ROYAI NATIONAT HOSPITAY FOR CONSUMPTION AND DISEASES OF THE CHEST, VENTYOR.

THE following necessarily brief observations which at the request of the distinguished Vice-President $I$ have the honour to bring before this great Congress form a purely clinical contribution to the therapeutics of pulmonary tuberculosis. They are derived from the comparative study, extending over a period of nearly 24 years, of some 10,000 carefully recorded cases, some in private practice, but for the most part in the Royal National Hospital for Consumption and Diseases of the Chest at Ventnor. Many of the patients have been under continuous observation for several years and others have presented themselves at varying intervals, thus affording opportunity for recognising changes in the physical signs and verifying progress or the reverse. The

17 Rivista di Patologia Nerrosa e Mentale, vol. ii., fasc. 2 (February, 1897, p. 72).

1 A paper read at the International Congress on Tuberculosis at Berlin, May 26th, 1899. 